(n)

trobertivier Journal of Nonlinear Mathematical Physics

\title{
On the nonexistence of Liouvillian first integrals for generalized Liénard polynomial differential systems
}

Guillaume Chèze, Thomas Cluzeau

To cite this article: Guillaume Chèze, Thomas Cluzeau (2013) On the nonexistence of Liouvillian first integrals for generalized Liénard polynomial differential systems, Journal of Nonlinear Mathematical Physics 20:4, 475-479, DOI:

https://doi.org/10.1080/14029251.2013.868260

To link to this article: https://doi.org/10.1080/14029251.2013.868260

Published online: 04 January 2021 


\title{
LETTER TO THE EDITOR
}

\section{On the nonexistence of Liouvillian first integrals for generalized Liénard polynomial differential systems}

\author{
Guillaume Chèze \\ Institut de Mathématiques de Toulouse; Université Paul Sabatier Toulouse 3; CNRS UMR 5219 \\ MIP Bât 1R3, 31062 TOULOUSE cedex 9, France \\ guillaume.cheze@math.univ-toulouse.fr \\ Thomas Cluzeau \\ Université de Limoges ; CNRS ; XLIM UMR 7252 ; DMI \\ 123 avenue Albert Thomas, 87060 LIMOGES cedex, France \\ thomas.cluzeau@xlim.fr
}

Received 8 June 2013

Accepted 18 September 2013

\begin{abstract}
We consider generalized Liénard polynomial differential systems of the form $\dot{x}=y, \quad \dot{y}=-g(x)-f(x) y$, with $f(x)$ and $g(x)$ two polynomials satisfying $\operatorname{deg}(g) \leq \operatorname{deg}(f)$. In their work, Llibre and Valls have shown that, except in some particular cases, such systems have no Liouvillian first integral. In this letter, we give a direct and shorter proof of this result.

Keywords: Polynomial vector fields, first integrals, invariant algebraic curves, Liénard polynomial differential systems
\end{abstract}

2000 Mathematics Subject Classification: 37K10, 34D30, 34C26

\section{Introduction}

We consider generalized Liénard polynomial differential systems of the form

$$
\left\{\begin{array}{l}
\dot{x}=y \\
\dot{y}=-g(x)-f(x) y
\end{array}\right.
$$

where $f(x)$ and $g(x)$ are two polynomials in the variable $x$ with complex or real coefficients and dot denotes the derivative with respect to the time variable $t$.

Liénard equations have been first introduced in [1] with $g(x)=c x$, where $c$ is a constant, in order to study the description of relaxation oscillations. They contain as a particular case, the wellknown van der Pol equations which correspond to $f(x)=\mu\left(x^{2}-1\right)$, where $\mu$ is a constant. In [2], Levinson and Smith have introduced a more general description of relaxation oscillations given by the generalized Liénard equations (1.1) considered in the present paper. Since then, generalized Liénard equations have been well studied. In particular, a lot of results have been published on limit cycles and periodic solutions of generalized Liénard equations: see for example [3] and references therein. 
An important and difficult issue in the study of polynomial vector fields $\dot{x}=A(x, y), \dot{y}=B(x, y)$, where $A$ and $B$ are two polynomials, consists in studying the existence of first integrals, that is, the existence of functions which level sets correspond to solutions of the vector field. The existence of first integrals is known to be related to the existence of invariant algebraic curves: for example, see Theorem 2.1 below.

In [4, 5], Odani shows that if $\operatorname{deg}(g) \leq \operatorname{deg}(f)$ and if we avoid some trivial situations, the generalized Liénard polynomial differential system (1.1) has no invariant algebraic curve and thus no elementary first integral, i.e., no first integral which is a finite composition of algebraic functions, exponentials and logarithms. Then, Żołądek follows this study by characterizing the existence of invariant algebraic curves of generalized Liénard equations for all values of $\operatorname{deg}(f)$ and $\operatorname{deg}(g)$, see [6].

More recently, in [7, 8], Llibre and Valls classify Liouvillian first integrals for Liénard polynomial differential systems of the form (1.1) in the case $\operatorname{deg}(g) \leq \operatorname{deg}(f)$. Roughly speaking, Liouvillian functions are functions which can be obtained by quadratures of elementary functions. They prove that, if we avoid some trivial situations, System (1.1) with $\operatorname{deg}(g) \leq \operatorname{deg}(f)$ has no Liouvillian first integral. The contribution of the present letter is to provide a direct and shorter proof of this result. Indeed, the approach proposed here avoids both the characterization of exponential factors and the recombination process used by Llibre and Valls, see Section 3.

\section{Preliminaries}

We recall here some definitions and theorems useful to understand the sequel.

Given two polynomials $A, B \in K[x, y]$, where $K=\mathbb{R}$ or $\mathbb{C}$, we consider a planar polynomial vector field

$$
\left\{\begin{array}{l}
\dot{x}=A(x, y) \\
\dot{y}=B(x, y)
\end{array}\right.
$$

and the associated derivation $\mathscr{D}=A(x, y) \partial_{x}+B(x, y) \partial_{y}$, where $\partial_{x}=\frac{\partial}{\partial x}$ and $\partial_{y}=\frac{\partial}{\partial y}$.

Definition 2.1. A polynomial $P \in \mathbb{C}[x, y]$ is a Darboux polynomial of (2.1) if there exists a polynomial $\Lambda \in \mathbb{C}[x, y]$ such that $\mathscr{D}(P)=\Lambda P$.

A function $F$ is a first integral of (2.1) if it satisfies $\mathscr{D}(F)=0$.

A function $F$ is a Liouvillian first integral of (2.1) if it is a first integral of (2.1) which is a Liouvillian function, i.e., belongs to a differential field extension of $K(x, y)$ obtained by successive adjunctions of integrals, exponentials of integrals, and algebraic extensions.

If $P$ is a Darboux polynomial, we can remark that the curve $P=0$ is composed of orbits of the differential system. Thus, Darboux polynomials define invariant algebraic curves which explains why there are also called invariant algebraic curves which is the terminology used in this paper.

A useful tool in the study of first integrals of differential systems is the integrating factor.

Definition 2.2. A function $R$ is an integrating factor of the polynomial vector field (2.1) if $\mathscr{D}(R)=-\left(\partial_{x}(A)+\partial_{y}(B)\right) R$. 
If $R$ is an integrating factor of (2.1), then we have $\partial_{x}(R A)=-\partial_{y}(R B)$. This means that the differential form $\omega=-R B \mathrm{~d} x+R A \mathrm{~d} y$ is closed. Thus, this form has locally a primitive which is a first integral of (2.1). This explains why integrating factors are important in the study of integrability of differential systems of the form (2.1). In [9], Prelle and Singer have given a structure theorem about integrating factors of polynomial differential systems with elementary first integrals. In [10], Singer gives the same kind of result for polynomial differential systems with Liouvillian first integrals. We recall here this latter result as it will be used in the following.

Theorem 2.1 (Singer). If System (2.1) has a Liouvillian first integral, then there exists an integrating factor of (2.1) of the form

$$
\exp \left(\frac{P}{Q}\right) \prod_{i} Q_{i}^{\lambda_{i}}
$$

where $Q$ and the $Q_{i}$ 's are invariant algebraic curves of (2.1), $P$ is polynomial in $\mathbb{C}[x, y]$ and the $\lambda_{i}$ 's are complex numbers.

Theorem 2.1 shows that Liouvillian first integrals are related to invariant algebraic curves. The statement used here is due to Christopher, see [11].

In the situation of the generalized Liénard polynomial differential system (1.1) considered here, the associated derivation is given by $\mathscr{D}_{L}=y \partial_{x}-(g(x)+f(x) y) \partial_{y}$. Using the particular form of this derivation, in [4], Odani proves the following result concerning invariant algebraic curves of (1.1).

Theorem 2.2 (Odani). If $\operatorname{deg}(g) \leq \operatorname{deg}(f), f(x) \neq 0, g(x) \neq 0$, and $g(x) \neq \alpha f(x)$ for a complex number $\alpha$, then the generalized Liénard polynomial differential system (1.1) has no invariant algebraic curve.

\section{Main result}

In $[7,8]$, the authors study the existence of Liouvillian first integrals of the Liénard polynomial differential system (1.1) when $\operatorname{deg}(g) \leq \operatorname{deg}(f)$. Their approach consists in first computing all exponential factors, namely, functions of the form $E=\exp (P / Q)$, where $P$ and $Q$ are polynomials in $\mathbb{C}[x, y]$ such that there exists a polynomial $\Gamma \in \mathbb{C}[x, y]$ with total degree at most $\max (\operatorname{deg}(A), \operatorname{deg}(B))-1$ satisfying $\mathscr{D}(E)=\Gamma E$, and then, trying to recombine them in order to get an integrating factor of the form given in Theorem 2.1. They prove that System (1.1) has no Liouvillian first integral under the hypotheses of Theorem 2.2. The purpose of this letter is to give a direct proof which avoids the recombination problem of exponential factors. Instead of computing exponential factors, we consider directly the equation defining an integrating factor. We take advantage of the fact that for the generalized Liénard polynomial differential system (1.1), we have the following particular form of the divergence: $\partial_{x}(A)+\partial_{y}(B)=-f(x)$. Then, using both Theorems 2.1 and 2.2, we reduce the problem to the study of polynomial solutions $P(x, y)$ of the equation $\mathscr{D}_{L}(P)=f(x)$.

The result can be stated as follows:

Theorem 3.1. Consider the generalized Lienard differential system (1.1) with the condition $\operatorname{deg}(g) \leq \operatorname{deg}(f)$. If we further assume $f(x) \neq 0, g(x) \neq 0$, and $g(x) \neq \alpha f(x)$ for a complex number $\alpha$, then System (1.1) has no Liouvillian first integral. 
This provides a complete classification of Liouvillian first integrals of the generalized Liénard differential system (1.1) with $\operatorname{deg}(g) \leq \operatorname{deg}(f)$ since we have:

(i) if $f(x)=0$, then (1.1) admits the first integral $H(x, y)=y^{2}+2 \int g(x) \mathrm{d} x$,

(ii) if $g(x)=0$, then (1.1) admits the first integral $H(x, y)=y+\int f(x) \mathrm{d} x$,

(iii) if $g(x)=\alpha f(x)$, where $\alpha$ is a complex number, then (1.1) admits the first integral $H(x, y)=y+\int f(x) \mathrm{d} x-\alpha \log (y+\alpha)$, see [5].

Note that we can check by straightforward calculations that the first integrals given above are correct, i.e., that in each case $\mathscr{D}_{L}(H)=0$.

We shall now give a direct proof of Theorem 3.1.

Proof. From Theorem 2.2, we know that under the hypotheses of Theorem 3.1, System (1.1) has no invariant algebraic curve. Consequently, Theorem 2.1 implies that, if (1.1) has a Liouvillian first integral, then there exists a polynomial $P \in \mathbb{C}[x, y]$ such that $\mathscr{D}_{L}(\exp (P))=f(x) \exp (P)$ which is equivalent to $\mathscr{D}_{L}(P)=f(x)$. The latter equality can be written:

$$
(\star) \quad y \partial_{x}(P(x, y))-(g(x)+f(x) y) \partial_{y}(P(x, y))=f(x) .
$$

The following strategy is close to what is done in a slightly different context in [4] to prove Theorem 2.2. We set $P(x, y)=\sum_{i=0}^{n} P_{i}(x) y^{i}$, with $P_{n}(x) \neq 0$ and we denote $P_{i}^{\prime}(x)=\partial_{x}\left(P_{i}(x)\right)$.

If $n=0$, then $(\star)$ yields $y P_{0}^{\prime}(x)=f(x)$ which is not possible since $f(x) \neq 0$.

If $n \geq 1$, then by identifying the coefficients in $y^{i}$ for $i=n+1, \ldots, 0$ in the left-hand side of Equation $(\star)$, we get the following system of equations for the $P_{i}$ 's:

$$
\left\{\begin{array}{cl}
P_{n}^{\prime}(x) & =0 \\
P_{n-1}^{\prime}(x) & =n f(x) P_{n}(x) \\
P_{n-2}^{\prime}(x) & =(n-1) f(x) P_{n-1}(x)+n g(x) P_{n}(x) \\
\vdots & \\
P_{n-j}^{\prime}(x) & =(n-j+1) f(x) P_{n-j+1}(x)+(n-j+2) g(x) P_{n-j+2}(x) \\
\vdots & \\
P_{1}^{\prime}(x) & =2 f(x) P_{2}(x)+3 g(x) P_{3}(x) \\
P_{0}^{\prime}(x) & =f(x) P_{1}(x)+2 g(x) P_{2}(x) \\
0 & =f(x)+g(x) P_{1}(x)
\end{array}\right.
$$

If $n=1$, then the equation $P_{1}^{\prime}(x)=0$ implies that $P_{1}(x)=\beta$ is a non zero constant, but then the last equation of (3.1) yields $f(x)=-\beta g(x)$ which does not satisfy the hypotheses of Theorem 3.1.

We now assume $n \geq 2$ and we are going to compute the degrees of polynomials $P_{i}(x)$ satisfying System (3.1).

The first equation of (3.1), $P_{n}^{\prime}(x)=0$, implies that $P_{n}(x)$ is a non zero constant. Then, from $P_{n-1}^{\prime}(x)=n f(x) P_{n}(x)$, we get that $P_{n-1}$ is a polynomial of degree $\operatorname{deg}(f)+1$. Using $\operatorname{deg}(g) \leq \operatorname{deg}(f)$, the next equation giving $P_{n-2}^{\prime}$ in terms of $f, g, P_{n}$ and $P_{n-1}$ implies $\operatorname{deg}\left(P_{n-2}\right)=2(\operatorname{deg}(f)+1)$. By an easy induction, we obtain:

$$
\operatorname{deg}\left(P_{n-j}\right)=j(\operatorname{deg}(f)+1), \quad j=0, \ldots, n .
$$

In particular, we have $\operatorname{deg}\left(P_{1}\right)=(n-1)(\operatorname{deg}(f)+1)$.

Now, we consider the last equation $f(x)=-g(x) P_{1}(x)$ of (3.1). Comparing the degrees in each side, 
we obtain $\operatorname{deg}(f)=\operatorname{deg}(g)+(n-1)(\operatorname{deg}(f)+1)$ which is impossible since $n \geq 2$. In conclusion, we have shown that, under the conditions of Theorem 3.1, Equation $(\star)$ has no polynomial solution. This implies that there does not exist a polynomial $P \in \mathbb{C}[x, y]$ such that $\mathscr{D}_{L}(\exp (P))=f(x) \exp (P)$ so that, finally, System (1.1) has no Liouvillian first integral.

\section{References}

[1] A. Liénard, Étude des oscillations entretenues, Revue générale de l'électricité 23(21) (1928) 901-912.

[2] N. Levinson and O. K. Smith, A general equation for relaxation oscillations, Duke Math. J. 9 (1942) 382-403.

[3] H. Giacomini and S. Neukirch, Number of limit cycles of the Liénard equation, Phys. Rev. E (3) 56(4) (1997) 3809-3813.

[4] K. Odani, The limit cycle of the van der Pol equation is not algebraic, J. Differential Equations 115(1) (1995) 146-152.

[5] K. Odani, The integration of polynomial Liénard systems by elementary functions, Differential Equations Dynam. Systems 5(3-4) (1997) 347-354, Planar nonlinear dynamical systems (Delft, 1995).

[6] H. Żoładek, Algebraic invariant curves for the Liénard equation, Trans. Amer. Math. Soc. 350(4) (1998) $1681-1701$.

[7] J. Llibre and C. Valls, Liouvillian first integrals for Liénard polynomial differential systems, Proc. Amer. Math. Soc. 138(9) (2010) 3229-3239.

[8] J. Llibre and C. Valls, Liouvillian first integrals for generalized Liénard polynomial differential systems, To appear in Adv. Nonlinear Stud., (2013).

[9] M. J. Prelle and M. F. Singer, Elementary first integrals of differential equations, Trans. Amer. Math. Soc. 279(1) (1983) 215-229.

[10] M. F. Singer, Liouvillian first integrals of differential equations, Trans. Amer. Math. Soc. 333(2) (1992) 673-688.

[11] C. Christopher, Liouvillian first integrals of second order polynomial differential equations, Electron. J. Differential Equations (1999) No. 49, 7 pp. (electronic). 